group was significantly lower with 337 responses per session. The nonimplanted control ss reflect the operant level of the apparatus with 63.4 responses per session. This data, then, supports the findings of other investigators that brain area is a major variable in rate of response for intracranial stimulation.

The data from Phase II also show a significant difference between brain areas. The hypothalamic implant group was again best with a mean of 79.8 percent of total responses made in the intervals during which the cue light was on, as compared to only 67.3 percent for the septal implant group. Data reported here indicate that locus of stimulation is an important factor in the equality of discrimination performance for intracranial stimulation.

Significant differences between brain areas are also shown in the results from Phase III. As in the other two phases, the hypothalamic implant group was significantly higher than the septal group. Ss in the hypothalamic group had a mean extinction score of 102.6 responses, while Ss in the septal group had a mean extinction score of 65.8. Nonimplanted control Ss had a mean of 40 responses during the final session.

It should be pointed out that discrimination training had no effect upon resistance to extinction. The two hypothalamic implants and the two septal implants which received training, but not discrimination training, did not differ from their group mates in extinction. This indicates that extinction scores were not inflated by the discrimination training, as might be expected in some cases.

It seems that the data obtained in Phase III, though

\section{Reply to Wechkin and Sackett}

by H. S. Pennypacker, James A. Horel, and Shirley A. Myers

In commenting on our previous paper (Myers, Horel, \& Pennypacker, 1965) Wechkin and Sackett raise two issues which evidently require clarification. The first, concerning the operant nature of the VR, is clearly definitional-they suggest that we have inadvertently identified as an operant VR a response which may in fact have been respondent. The second relates to the origin of vocal behavior and is at best, speculative and interpretational.

We have used the conventional definition of the word operant defined originally by Skinner (1938, p. 21) as "... an identifiable part of behavior of which it may be said not that no stimulus can be found that will elicit it (there may be a respondent, the response of which has the same topography) but that no correlated stimulus can be detected upon occasions when it is observed to occur." From the same reference we find respondent defined as "the kind of behavior correlated with specific eliciting stimuli..."

In our procedure one of a number of existing VRs is selectively reinforced and as typically happens in similar situations, the response increases in frequency based on only seven Ss in each of the two brain areas, have especially important implications because of the inconsistencies and contradictions found in previous studies investigating extinction with intracranial stimulation. Howarth \& Deutsch (1962) reported rapid extinction as a function of time, for instance, yet Herburg (1963) could not predict the effect of manipulating certain variables upon extinction with Deutsch's theory. These apparent inconsistencies could be easily reconciled by systematic demonstrations that the area stimulated is a major factor in the extinction curves for intracranial stimulation. The present investigation, though of only a preliminary nature, suggests that brain area is such a determiner.

Curves for all three phases are presented in the figure.

\section{References}

Herburg, L. J. Determinants of electrical self-stimulation. $J$. comp physiol. Psychol., 56, 686-690, 1963.

Howarth, C. I., \& Deutsch, J. A. Drive decay: The cause of fast extinction of habits learned for brain stimulation. Science, 137 . $35-36,1962$

olds, J. A preliminary mapping of electrical reinforcing effects in the rat brain. J. comp. phusiol. Psychol., 49, 3, 281-286, 1956.

Olds, J. Approach-avoidance dissociations in the rat brain. Amer. J. Physiol., 199, 965-968, 1960

Olds, M. F., \& Olds, J. Approach-avoidance analysis of rat diencephalon. J. comp. Neurol., 120, 2, 259-295, 1963.

\section{Notes}

1. Part of this research was reported at the 1965 meeting of the Rocky Mountain Psychological Association, Denver, Colorado.

2. Now at Kansas State University.

until a stable level of responding is achieved. Clearly, the food does not elicit the response, it follows it and "anticipation" of the food is clearly not an identifiable stimulus. Moreover, attention should be called to the fact that the vocal behavior in question was maintained on an FR 3 schedule; the classical conditioning literature is hardly replete with examples of responses maintained over long periods on a $33 \%$ reinforcement schedule (Kimble, 1964).

With regard to the origin of vocal behavior, our report simply did not address itself to that question. While it is true that modifying a response by operant methods in the laboratory does not prove that similar behavioral alterations in the natural setting are due to operant conditioning, neither does it preclude such a possibility. Nor does it preclude the unrelated possibility that the behavior initially functioned as a respondent; e.g., the human blink response appears to undergo such modification in the case of the unilateral lid closure which is a significant component of the courtship pattern.

In any case, even if the VR is a food call, we would ask what it serves the animal. Prior to conditioning, the response probably had some functional significance for the animal, but whether it was a food call or mating 


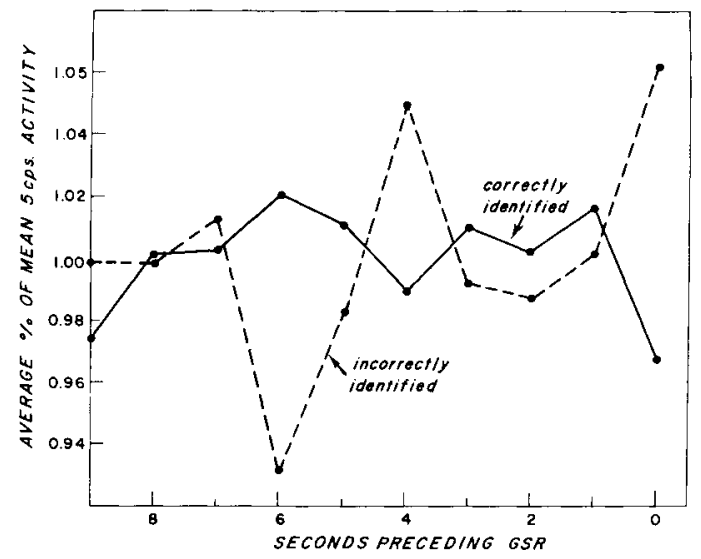

Fig. 1. EEG patterns preceding correctly and incorrectly identified GSRs. Averages of individual patterns for $10 \mathrm{ss}$.

GSRs ( rho $=.59, \mathrm{p}<.05$ ). Thus differential $5 \mathrm{cps}$ EEG activity preceding a GSR is related to its discriminability, with better discriminators showing a greater EEG differential.

\section{Discussion}

The EEG pattern preceding a GSR indicates whether or not it will be discriminated. Since the EEG activity precedes the GSR, the activity cannot be due to afferent feedback from the autonomic change. Thus it is clear that such feedback is not the sole determinant of GSR discriminability.

There are, however, two possible interpretations of the EEG patterns. One is that the pattern preceding discriminated GSRs represents a central event which produces the GSR and in turn serves as a cue for its discrimination. This event would then be discriminable whether or not a GSR were present. This interpretation is in accord with the introspections of the Ss. Good discriminators reported using cues such as "When a new thought popped into my mind after I had been concentrating on one thing for a few seconds," or "When I thought of emotional experiences."

The other interpretation is that the event indicated by the EEG patterns only modifies the ability to discriminate autonomic feedback from the periphery. In this case, GSR discrimination would require the simultaneous occurrence of central and peripheral events. The preceding central events would not necessarily be discriminable by themselves. A decisive experiment would entail testing for GSR discrimination after deafferentation.

Although a consistent relationship between alpha rhythm alerting responses and GSR discrimination was not found, the importance of the $5 \mathrm{cps}$ activity is consistent with Walter's (1953) view regarding theta rhythm as an indicator of emotion.

\section{References}

Becker, H. C., Mickle, W. A., \& Heath, R. G. A variable frequency variable selectivity filter for electroencephalography. $E E G$ clin Neurophysiol., 1958, 10, 731-735.

Crider, A., Shapiro, D., \& Tursky, B. Reinforcement of spontaneous electrodermal activity. J. comp. physiol. Psychol, in press Mandler, G.. \& Kahn, M. Discrimination of changes in heart rate: Two unsuccessful attempts. $J$. exp. Anal. Behav., 1960, 3, 21-25. Tursky, B. Integrators as measuring devices of bioelectric output. Clin. Pharmacol. Therap., 1964, 5, 887-892.

Walter, W. G. The living brain. New York: Norton, 1953.

\section{Notes}

1. The work forms part of an honors thesis in the Department of Psychology at Harvard College. It was supervised by Drs. David Shapiro and Andrew Crider and Mr. Bernard Tursky at the Massachusetts Mental Health Center. The author is grateful to them for their critical advice and support. Financial assistance was provided by NIMH Grant MH-08853-02 and by ONR Contract Nonr$1866(43)$.

2. The form of the two curves may result from the tendency of correctly and incorrectly identified GSRs to fall at different phases of periodic fluctuations of $5 \mathrm{cps}$ activity.

\section{Continued from page 254 .}

call is totally irrelevant. Presumably, any response conditioned by operant methods had some significance to the animal prior to its selective reinforcement. If it is used somehow in obtaining food it is an operant already and we have simply submitted it to further operant conditioning. If it is ordinarily elicited simply in response to food, then we have shown that this response can be operantly controlled.

We are happy to see that our critics support our only conclusion, viz. operant conditioning of the VR of nonhuman primates can be achieved. It is a point on which there has not been unanimous agreement among authorities prior to our experiment (Lilly, 1960; Andrew, 1962).

With our statement on acquisition of VRs back in the context from which it was removed, it is clear that we were not suggesting that all VRs of all animals are acquired in this way. The point is that there are now a number of experiments demonstrating operant control of VRs in nonhumans. What remains to be demonstrated is whether or not this operant conditioning is different in any fundamental way from the operant conditioning that is postulated as being important in the acquisition of human verbal behavior.

\section{References}

Andrew, R. J. Evolution of intelligence and vocal mimicking. Science, 1962, 137, 585-589

Kimble, G. A. The problem of definition. In A. W. Melton (Ed.) Categories of human learning. New York: Academic Press, 1964.

Lilly, J. C. Third conference on the central nervous system and behavior. In M. A. B. Brazier (Ed.), New York Josiah Macy, Jr. Foundation, 1960

Skinner, B. F. The behavior of organisms. An experimental analysis. New York: Appelton-Century-Crofts, 1938. 